\title{
Der Europäische Auswärtige Dienst und die Organisation europäischer Außen- und Entwicklungshilfe: von institutionellen Dissonanzen zur dienstübergreifenden Harmonie?
}

\author{
Isabelle Tannous*
}

Der Vertrag von Lissabon hatte die künftige Organisation der europäischen Außen- und Entwicklungshilfe im neuen institutionellen Gefüge zunächst einmal offen gelassen. Gleichzeitig sind mit dem Europäischen Auswärtigen Dienst (EAD) hohe Erwartungen verbunden worden: So sollen die europäischen Außenbeziehungen kohärenter, wirksamer und sichtbarer werden, um die Gestaltungsmöglichkeiten der Europäischen Union auf globaler Ebene zu stärken. Wer die europäische Außenpolitik in den letzten Jahrzehnten verfolgt hat, weiß um die Fallhöhe dieser politischen Zielmarken - insbesondere in Anbetracht der wenig konkreten Vorgaben im Vertragswerk. Diese sollten erst nach dessen Inkrafttreten am 1. Dezember 2009 mit der Einpassung des EAD in das institutionelle Gefüge mit Leben gefüllt werden. Im Mittelpunkt der Kompetenzstreitigkeiten rund um den EAD stand - und steht dabei die Außen- und Entwicklungshilfe der Europäischen Union. Insbesondere beim sensiblen Thema der Arbeitsteilung zwischen dem Rat der Europäischen Union beziehungsweise EAD und der Europäischen Kommission werden die Karten neu gemischt. Neben der Neuordnung der politischen Verantwortlichkeiten sowie der Entscheidungsstrukturen und -verfahren steht dabei die Frage im Raum, welche Folgen sich aus den umfangreichen Änderungen für die strategische Ausrichtung der EU-Außenbeziehungen und den politischen Stellenwert der Außen- und Entwicklungshilfe insgesamt ergeben.

\section{Würdigung der entwicklungspolitischen Zielsetzung in den primärrechtlichen Vorgaben von Lissabon}

Die EU-Entwicklungszusammenarbeit umfasst längst nicht mehr nur die aus der Kolonialvergangenheit einiger EU-Mitgliedstaaten entstandene Zusammenarbeit mit den 79 Staaten Afrikas, der Karibik und des Pazifiks (AKP), deren Vertragsgrundlage das Abkommen von Cotonou bildet. Heute unterhält die Europäische Union Beziehungen mit Partnerstaaten in allen Regionen der Welt und ist mit über 50 Prozent der öffentlichen Entwicklungshilfe der größte Geber weltweit. Nach Aufdeckung der Korruptions- und Betrugsfälle der SanterKommission Ende der 1990er Jahre hat ein umfangreicher Reformprozess der Strukturen und Verfahren der EU-Entwicklungszusammenarbeit stattgefunden, dessen konzeptionelle Neuorientierung der „Europäische Konsens über die Entwicklungspolitik“1 (2005) und

\footnotetext{
* Isabelle Tannous, M.A., Fachinformation Europa, EU, EU-Integration, EU-Außenbeziehungen, Stiftung Wissenschaft und Politik, Berlin.

1 Gemeinsame Erklärung des Rates und der im Rat vereinigten Vertreter der Regierungen der Mitgliedstaaten, des Europäischen Parlaments und der Kommission zur Entwicklungspolitik der Europäischen Union: Der Europäische Konsens über die Entwicklungspolitik, in: Amtsblatt der EU, Nr. C 46 vom 24. Februar 2006, S. 1-18.
} 
jüngst die „Agenda für Wandel“2 (2011) wiedergeben. Mit der fortschreitenden Auflösung der EU-Zusammenarbeit mit den AKP-Staaten, der Verankerung der Armutsbekämpfung als vorrangigem Ziel der EU-Entwicklungspolitik, aber auch dem Bedeutungszuwachs der BRICS-Staaten auf der globalen Entwicklungsagenda und den Umbrüchen im Nahen und Mittleren Osten haben sich Charakter und Koordinaten der europäischen Außen- und Entwicklungshilfe verschoben. ${ }^{3}$ Während die Folgen dieser Dynamiken für die Europäische Union in den letzten Jahren unter dem technokratisch anmutenden Alltagsgeschäft noch verborgen blieben, sprechen die institutionellen Veränderungen rund um die Schaffung des Hohen Vertreters der Union für Außen- und Sicherheitspolitik und insbesondere den EAD nun eine deutlichere Sprache. Der damit einhergehende Wandel wird gleichermaßen als Chance und als Gefahr für eine eigenständige EU-Entwicklungszusammenarbeit wahrgenommen. ${ }^{4}$ Da ist einerseits von einer verbesserten Kohärenz, mehr Transparenz, einer größeren Wirksamkeit der Maßnahmen und neuen Handlungsräumen für eine proaktive Entwicklungszusammenarbeit die Rede, gleichzeitig wird eine Vertiefung der ohnehin schon offen zutage liegenden institutionellen Gräben befürchtet und es bleibt die Besorgnis der Unterordnung genuin entwicklungspolitischer Ziele - in erster Linie also der Armutsbekämpfung - unter ein vom Rat dominiertes, kurzfristiges Krisenmanagement.

Diese Grundsatzfragen standen bereits während des Konvents zur Zukunft der Europäischen Union und bei der Ausarbeitung des Vertrags von Lissabon im Raum. Ihre Brisanz offenbarte sich schließlich eruptiv bei der organisatorischen Konkretisierung der Vorgaben des Vertrags von Lissabon rund um den EAD. Dabei hat sich auf den ersten Blick mit dem durch den Vertrag von Lissabon neu geschaffenen Rechtsrahmen der Europäischen Union zunächst einmal wenig im Bereich der Außen- und Entwicklungshilfe verändert: Die Nachbarschaftspolitik erhält einen eigenen Artikel, ${ }^{5}$ die EU-Entwicklungszusammenarbeit bleibt weiterhin eigenständiges Politikfeld und wird gleichzeitig zum integralen Bestandteil des gesamten auswärtigen Handelns. ${ }^{6}$ Auch im Vertrag von Lissabon bleibt das Hauptziel der EU-Politik auf dem Gebiet der Entwicklungszusammenarbeit weiterhin die Bekämpfung und auf längere Sicht die Beseitigung von Armut. Neben diesem Bekenntnis zu den Millenniumsentwicklungszielen - die Armutsbekämpfung wird als Zielvorgabe nun sogar dem ge-

2 Europäische Kommission: Mitteilung der Kommission an das Europäische Parlament, den Rat, den Europäischen Wirtschafts- und Sozialausschuss und den Ausschuss der Regionen: Für eine EU-Entwicklungspolitik mit größerer Wirkung: Agenda für den Wandel, KOM (2011) 637.

3 Siehe Isabelle Tannous: Die Entwicklungszusammenarbeit und humanitäre Hilfe der Europäischen Union, in: Werner Weidenfeld (Hrsg.): Die Europäische Union. Politisches System und Politikbereiche, Bonn 2008, S. 434-454; sowie die Beiträge zur Entwicklungszusammenarbeit und humanitären Hilfe in Werner Weidenfeld/ Wolfgang Wessels (Hrsg.): Jahrbuch der Europäischen Integration, Bonn/Baden-Baden, verschiedene Jahrgänge.

4 Vgl. beispielsweise European Think-Tanks Group: New Challenges, New Beginnings. Next Steps in European Development Cooperation, February 2010; Overseas Development Institute/European Centre for Development Policy Management/Fride/Deutsches Institut für Entwicklungspolitik: Development-proofing the European External Action Service, European Think-Tanks Group Policy Brief, June 2010; Paul Engel: On development policy, the EU's External Action Service is looking vulnerable, in: europesworld.org, 10.3.2011; Toby Vogel: The difficulties of dividing up development responsibilities, in: European Voice, 15.7.2010.

5 Art. 8 Vertrag über die Europäische Union (EUV).

6 Art. 208 bis 211 Vertrag über die Arbeitsweise der Europäischen Union (AEUV). Vgl. exemplarisch Jeske van Seters/Henrike Klavert: EU development cooperation after the Lisbon Treaty. People, institutions and global trends ECDPM, Discussion Paper No. 123, December 2011; Morten Broberg: What Is the Direction for the EU's Development Cooperation after Lisbon? A Legal Examination, in: European Foreign Affairs Review 4/2011, S. 539-557; Mark Furness: The Lisbon treaty, the European external action service and the reshaping of EU development policy, in: Stefan Gänzle/Sven Grimm/Davina Makhan (Hrsg.): The European Union and global development: An 'Enlightened Superpower' in the Making?, Houndmills 2012, S. 74-93. 
samten auswärtigen Handeln vorangestellt ${ }^{7}$ - bleibt die parallele Zuständigkeit der Mitgliedstaaten und der Union bestehen, die sich auf dem Gebiet der Entwicklungszusammenarbeit lediglich ergänzen beziehungsweise nun sogar verstärken sollen. Außerdem soll die Union bei der Durchführung aller politischer Maßnahmen, die sich auf die Entwicklungsländer auswirken können, den Zielen der Entwicklungszusammenarbeit Rechnung tragen, so wie es bereits mit der Agenda für mehr Politikkohärenz im Interesse der Entwicklung angelegt ist (Policy Coherence for Development, PCD) ${ }^{8}$ Die Lissabonner Reform übernimmt neben dieser PCD-Agenda auch den Besitzstand des Europäischen Konsens für die Entwicklungspolitik (2005) und des „Europäischen Konsens über die humanitäre Hilfe“9 (2007) und überträgt somit in erster Linie die jüngsten Entwicklungen zwischen den Vertragsreformen in das Primärrecht.

Die vertragsrechtlich fixierten Koordinaten der europäischen Entwicklungszusammenarbeit bleiben somit im Großen und Ganzen bestehen - von einer bloßen Neunummerierung der bisherigen Verträge kann hingegen keine Rede sein: Durch die weitreichenden Änderungen der gesamten EU-Außenbeziehungen wird auch im Bereich der Außen- und Entwicklungshilfe zusammengelegt und neu verteilt. Aufgrund der bestehenden Unschärfe der Zuständigkeiten zwischen EAD und Kommission bei der europäischen Außen- und Entwicklungshilfe hatte dieser Prozess auch die Grundsatzfragen über die Zukunft der EU-Entwicklungszusammenarbeit neu aufgeworfen - und dies in einem Ausmaß, das möglicherweise einen grundlegenden Wandel der gesamten EU-Entwicklungszusammenarbeit vorantreibt.

\section{Der Streit um die Außen- und Entwicklungshilfe bei der Gründung des EAD}

Bevor der EAD überhaupt seine Arbeit aufnehmen konnte, galt es zunächst einmal die institutionellen und personellen Koordinaten zu klären. Schließlich war es den Staats- und Regierungschefs sowohl im Konvent als auch während der Verhandlungen über den Vertrag von Lissabon bei einer ganzen Reihe politisch umstrittener und organisatorisch komplexer Fragen nicht gelungen, eine gemeinsame Linie zu finden. ${ }^{10}$ Der Vertrag gab insbesondere mit Art. 27 EUV zum EAD und Art. 221 AEUV zu den EU-Delegationen lediglich ein grobes Gerüst vor. In welcher Form der EAD der Hohen Vertreterin zuarbeiten sollte, wurde genauso wenig näher spezifiziert, wie die Art und Weise seiner Zusammensetzung und die Modi der Zusammenarbeit mit den diplomatischen Diensten der Mitgliedstaaten sowie der Europäischen Kommission. Bündelt man die offen gebliebenen Fragen, so bleibt im Kern die zentrale Frage: Wie wird künftig die Strategie- und Programmplanung der Außen- und Entwicklungshilfe zwischen dem Dienst und der Europäischen Kommission zu gestalten sein?

7 Art. 21 EUV.

8 Siehe Europäische Kommission: Commission Staff Working Paper. EU 2011 Report on Policy Coherence for Development, SEC (2011) 1627; Confederation for Cooperation of Relief and Development NGOs (Concord): Spotlight on EU Policy Coherence for Development. A Lisbon Treaty provision - A Human Rights obligation, Report 2011.

9 Gemeinsame Erklärung des Rates und der im Rat vereinigten Vertreter der Regierungen der Mitgliedstaaten, des Europäischen Parlaments und der Europäischen Kommission (2008/C 25/01). Europäischer Konsens über die humanitäre Hilfe, in: Amtsblatt der EU, Nr. C 25 vom 30. Januar 2008, S. 1-12.

10 Vgl. Julia Lieb/Martin Kremer: Der Aufbau des Europäischen Auswärtigen Dienstes: Stand und Perspektiven, in: integration 3/2010, S. 195-208, hier S. 206-207. 
Dies ist angesichts der Höhe der für die Außen- und Entwicklungshilfe bereitgestellten Mittel keine unwichtige Frage, wie bereits der Blick in den EU-Haushalt zeigt. ${ }^{11}$ Der Gesamthaushaltsplan 2012 - von dem der von den Mitgliedstaaten separat gespeiste Europäische Entwicklungsfonds (EEF) sogar gänzlich ausgenommen ist ${ }^{12}$ - weist in Rubrik 4 , Globales Europa' allein für das Finanzierungsinstrument für die Entwicklungszusammenarbeit (DCI) 27,6 Prozent der vorgesehenen Mittel aus. Nimmt man noch die Mittel des Europäischen Nachbarschafts- und Partnerschaftsinstruments (ENPI) mit 24,4 Prozent hinzu, so umfassen diese beiden großen Instrumente gemeinsam über 50 Prozent der bereitgestellten Mittel. ${ }^{13}$ Wird noch der nicht im EU-Haushalt geführte EEF mit einbezogen, so machen die drei mittelstärksten Außen- und Entwicklungshilfeinstrumente EEF, DCI und ENPI gemeinsam sogar insgesamt 9 Prozent des gesamten EU-Haushalts aus.

Entstanden aus den Sonderbeziehungen einiger Mitgliedstaaten zu den ehemaligen Kolonien und mit der Selbstverpflichtung auf die Millenniumsentwicklungsziele nach neuer Legitimation strebend, bringt die EU-Entwicklungszusammenarbeit einiges an integrationspolitischem Gepäck mit sich. Dies wirkt sich nachteilig auf Wirksamkeit und Kohärenz aus, entsprechend ist die Diskussion um die ,3 Ks ${ }^{6}$ - Koordination, Kohärenz, Komplementarität - beinahe so alt wie die europäische Entwicklungszusammenarbeit selbst. ${ }^{14}$ Die daraus resultierenden Reibungsflächen in mindestens drei Dimensionen sind bekannt: die unterschiedlichen mitgliedstaatlichen Ansätze bei den nationalen Entwicklungspolitiken sowie bei der Neuausrichtung einer gemeinsamen EU-Politik, das Zusammenspiel der europäischen Institutionen und die Entwicklungszusammenarbeit als transversale Aufgabe. Mit der Umstrukturierung rund um den EAD bot sich damit entsprechend die Gelegenheit, die europäische Außen- und Entwicklungshilfe durch eine kohärentere Anbindung an andere Politikbereiche zu profilieren.

Grundsätzlich sind die EU-Mitgliedstaaten aber auch nach dem Inkrafttreten des Vertrags von Lissabon nur begrenzt bereit, ihre entwicklungspolitischen Initiativen von der Europäischen Kommission koordinieren oder gar kontrollieren zu lassen. Es liegt nicht in ihrem Interesse auf Druck aus Brüssel hin ihren Hilfsverpflichtungen nachzukommen oder, wie im freiwilligen EU-Verhaltenskodex zu Komplementarität und Arbeitsteilung in der Entwicklungspolitik von $2007^{15}$ vereinbart, ihre Länderprioritäten untereinander zu koordinieren. Mit der Gründung des EAD eröffnete sich nun für diejenigen EU-Mitgliedstaaten, die weiterhin daran interessiert waren im Bereich der Außen- und Entwicklungshilfe souveräne Entscheidungen zu treffen, die Möglichkeit, strategische Kompetenzen, die über die Verwaltung hinausgingen, im EAD und damit (wieder) in einer den Mitgliedstaaten näher stehenden Institution anzusiedeln. Dies betraf neben dem erneuten Verzicht auf eine ,Budge-

11 Im Jahr 2011 entsprachen 92 Prozent der Außen- und Entwicklungshilfe der ODA-Klassifizierung. Auch wenn damit der Löwenanteil der europäischen Außen- und Entwicklungshilfe - DCI (beinahe 100 Prozent ODA), ENPI (95 Prozent ODA) und IPA (90 Prozent ODA) - als öffentliche Entwicklungshilfe klassifiziert werden kann, ist ein Großteil dieser Mittel für Staaten mit mittlerem Einkommen vorgesehen.

12 Der Europäische Entwicklungsfonds (EEF) verbleibt auch nach den Reformen durch den Vertrag von Lissabon außerhalb des EU-Haushalts, er wurde jedoch in der Folge an die Laufzeit und Verfahren des EU-Finanzrahmens angepasst.

13 Für das Instrument für Heranführungshilfe (IPA) sind 20,2 Prozent veranschlagt, während auf die humanitäre Hilfe 8,5 Prozent und auf die GASP lediglich 4,2 Prozent der Mittel entfallen. Eigene Berechnungen (gerundet) auf Basis der Zahlen aus: Europäische Kommission: EU-Haushalt 2012, Luxemburg 2012.

14 Siehe exemplarisch Maurizio Carbone: Mission Impossible: the European Union and Policy Coherence for Development, in: Journal of European Integration 3/2008, S. 323-342.

15 Europäische Kommission: Mitteilung der Kommission an den Rat und das Europäische Parlament - EU-Verhaltenskodex im Hinblick auf die Arbeitsteilung im Bereich der Entwicklungspolitik, KOM (2007) 72. 
tierung' des EEF auch die beiden von der Kommission verwalteten wichtigsten Außenhilfeinstrumente DCI und ENPI. Den Mitgliedstaaten, die mit ihrer Entwicklungszusammenarbeit selbst Flagge zeigen beziehungsweise das entwicklungspolitische Engagement enger mit der Außenpolitik verzahnen wollen, steht die Europäische Kommission als eigenständiger Akteur mit ihren weitreichenden Kompetenzen in der Nachbarschafts- und Entwicklungspolitik gegenüber. Das Europäische Parlament und die Kommission befürworten wie diejenigen EU-Mitgliedstaaten, die der globalen Entwicklungsagenda besonders verpflichtet sind, ,mehr Europa“ in diesem Feld.

Vor diesem Hintergrund konnte es also kaum überraschen, dass sich der Streit über die Gestalt des EAD - und damit auch die künftige Ausrichtung der europäischen Außenbeziehungen - gleich bei Catherine Ashtons erstem Strategiepapier vom 25. März 2010 am Thema der Außen- und Entwicklungshilfe entzündete. ${ }^{16}$ Ashton machte bei den Verhandlungen zur Gründung des EAD frühzeitig deutlich, dass sie die Außen- und Entwicklungshilfe als einen integralen Bestandteil des Werkzeugkastens der Europäischen Union betrachtet, genauer, dass sie ,im Herzen des Auswärtigen Handelns der Europäischen Union“17 liege. Zum Auftakt der Verhandlungen skizzierte sie den EAD im März 2010 als eine einmalige Gelegenheit, für die Kohärenz des auswärtigen Handelns der Union zu sorgen und das vorhandene Instrumentarium - von den wirtschaftlichen und politischen Instrumenten über die Entwicklungszusammenarbeit bis hin zu den Krisenmanagementkapazitäten - unter einer einheitlichen politischen Strategie zusammenzuführen.

Bei der Zuständigkeitsverteilung zwischen dem künftigen Dienst und der Europäischen Kommission schlug Ashton für die Programmplanung der wichtigsten Außenhilfeinstrumente unterschiedliche Szenarien vor: Diese reichten, erstens, von der Übertragung des gesamten Planungszyklus an den EAD, über, zweitens, ein arbeitsteiliges Konzept unter Federführung der Kommission, bis zu, drittens, einer regionalen Aufgabenteilung, bei der sich die Kommission um Afrika und der EAD um Asien und Lateinamerika kümmern sollte. Anders als bei der Nachbarschaftspolitik wollte Ashton die Vergabe der Entwicklungshilfe nicht allein der Kommission überlassen, entsprechend forderte sie für den EAD die finanzielle Planungshoheit für fast alle großen Entwicklungsfonds. ${ }^{18} \mathrm{Ihr}$ Entwurf stand tendenziell der Haltung der EU-Außenminister näher, griff aber auch andere im Vorfeld diskutierte Szenarien auf. Während die Mitgliedstaaten den Führungsanspruch des EAD in - mindestens - den ersten beiden Phasen des Programmierungszyklus gesehen (und damit die strategische Zuständigkeit über die Mittelzuweisung und die Länder- und Regionalen Strategiepapiere) und der Kommission dann die nachfolgenden Phasen zugedacht hatten (wie die Nationalen und Regionalen Richtprogramme, die Jahresaktionsprogramme sowie die Implementierung

16 Draft Council decision establishing the organisation and functioning of the European External Action Service, 25. März 2010.

17 Vgl. Catherine Ashton, High Representative/Vice President: Speech to the European Parliament's foreign affairs committee European Parliament Brussels, 23. März 2010, SPEECH/10/120 (eigene Übersetzung).

18 Siehe auch Steven Blockmans/Simon Duke: The Lisbon Treaty stipulations on Development Cooperation and the Council Decision of 25 March 2010 (Draft) establishing the organisation and the functioning of the European External Action Service, European Institute of Public Administration: EIPA Working Paper 2010/W/01; Nikolas Busse: Ein Auswärtiger Dienst für die EU, in: Frankfurter Allgemeine Zeitung, 1.3.2010, S. 2; Leigh Phillips: Member states and EU commission clash over diplomatic service, in: euobserver.com, 2.3.2010; Doris Simon: Europas jüngstes Kind. Der Streit um Ausbau des Auswärtigen Dienstes der EU, in: Deutschlandfunk, 26.3.2010. 
und Evaluierung), hatte die Kommission statt solch einer horizontalen Aufteilung der Zuständigkeiten eine vertikale Teilung entlang geografischer Grenzen favorisiert. ${ }^{19}$

Erwartungsgemäß rief Ashtons Entwurf sowohl einige EU-Mitgliedstaaten wie auch die Kommission auf den Plan. Während die Mitgliedstaaten kritisierten, die Kommission würde weiterhin Teile der strategischen Planung übernehmen, wertete die Kommission Ashtons Vorlage dagegen als direkten Angriff auf ihre über Jahrzehnte hinweg erworbenen Kompetenzen. ${ }^{20}$ Sie sah sich in ihren Rechten beschnitten und fürchtete zu einer bloßen Exekutivagentur des EAD degradiert zu werden. Darauf bedacht, möglichst keine Befugnisse an den EAD zu verlieren, hatte sich die Kommission entsprechend frühzeitig vehement dafür eingesetzt, weiterhin für die Bereiche Entwicklung und Nachbarschaft - und damit die wichtigsten Außenhilfeinstrumente - verantwortlich zu zeichnen.

Nachdem die Staats- und Regierungschefs dem Vorschlag Ashtons am 26. April 2010 mit nur wenigen Änderungen zugestimmt hatten, ${ }^{21}$ erhielt die Kommission intensive Schützenhilfe aus dem Europäischen Parlament. Dieses war im Gegensatz zu den Mitgliedstaaten und der Kommission nicht in die Vorarbeiten zur Ausarbeitung von Ashtons Entwurf involviert, hatte sich aber mit dem ,Non-paper' von Elmar Brok und Guy Verhofstadt bereits vor Offenlegung des Entwurfs für eine enge Anbindung des EAD an die Kommission eingesetzt. ${ }^{22}$ Das Europäische Parlament, das insbesondere unter Federführung des Vorsitzenden des Ausschusses für Auswärtige Angelegenheiten Brok die Entscheidungen rund um den EAD im Paket verhandelte, ${ }^{23}$ nutzte die Gelegenheit, über den Hebel der Haushaltskontrolle die Kommission gegenüber dem EAD zu stärken und den eigenen Einflussbereich auszuweiten. Es nahm den vom sogenannten Quadrilog in Madrid am 21. Juni 2010 erzielten Kompromiss schließlich am 8. Juli 2010 mit großer Mehrheit an. ${ }^{24}$ Nach Monaten langwieriger Verhandlungen konnte so eine politische Einigung erzielt werden, die eine Arbeitsaufnahme des EAD zum Jahreswechsel 2010/2011 schließlich ein Jahr nach Inkrafttreten des Vertrags von Lissabon erlaubte.

\section{Die Außen- und Entwicklungshilfe auf den Agenden von EAD und Generaldirektion EuropeAid - Entwicklung und Zusammenarbeit (GD DEVCO)}

Mit dem EAD-Gründungsbeschluss vom 26. Juli 2010 ist es schließlich gelungen, wichtige Grundsatzfragen zu klären. ${ }^{25}$ Wie so häufig, kam allerdings auch dieser Beschluss letzt-

19 Vgl. Andrew Rettman: EU capitals defend Ashton's powers in diplomatic corps, in: euobserver.com, 22.3.2010; siehe auch Furness: The Lisbon treaty, 2012.

20 Vgl. Toby Vogel: Turf war continues over EU's diplomatic corps, in: European Voice, 11.3.2010.

21 Rat der Europäischen Union: Entwurf eines Beschlusses des Rates über die Organisation und die Arbeitsweise des Europäischen Auswärtigen Dienstes, Dok. 8029/10.

22 Proposal for the establishment of the EEAS, Working document by Elmar Brok (AFET), and Guy Verhofstadt (AFCO), rapporteurs on EEAS, 6.4.2010 beziehungsweise ergänzte Version 20.4.2010.

23 Europäisches Parlament: Bericht über den Vorschlag für einen Beschluss des Rates über die Organisation und die Arbeitsweise des Europäischen Auswärtigen Dienstes (08029/2010 - C7-0090/2010 - 2010/0816(CNS)), Ausschuss für auswärtige Angelegenheiten, Berichterstatter: Elmar Brok, 6.7.2010, A7-0228/2010.

24 Diesem gehörten die Hohe Repräsentantin, die EU-Kommission, die spanische EU-Präsidentschaft und eine informelle Parlamentsdelegation, besetzt mit Abgeordneten der drei großen Fraktionen EVP, S\&D und ALDE, an. Vgl. beispielsweise European Policy Centre/Egmont - The Royal Institute for International Relations/Centre for European Policy Studies: The Treaty of Lisbon: A Second Look at the Institutional Innovations, Joint Study, September 2010; Leendert Erkelens/Steven Blockmans: Setting up the European External Action Service. An institutional act of balance, Centre for the Law of EU External Relations: CLEER Working Papers 2012/1.

25 Beschluss des Rates vom 26. Juli 2010 über die Organisation und die Arbeitsweise des Europäischen Auswärtigen Dienstes (2010/427/EU), in: Amtsblatt der EU, Nr. L 201 vom 3. August 2010, S. 30-40. 
lich nur durch den Rückgriff auf vage Formulierungen zustande, die den beteiligten Akteuren genügend Interpretationsspielräume einräumten. Insbesondere bei der Organisation der europäischen Außen- und Entwicklungshilfe bedurfte es auch nach dem EAD-Gründungsbeschluss einer Feinjustierung. Deren Gegenstand sollte insbesondere sein, wie die Zuordnung der Zuständigkeiten und die Weisungsbefugnis in der Praxis aussehen sollten. Die Hohe Vertreterin Catherine Ashton und Entwicklungskommissar Andris Piebalgs kündigten zu diesem Zweck gleich im Anschluss an den Gründungsbeschluss die Ausarbeitung einer neuen dienstübergreifenden Vereinbarung an. ${ }^{26}$ Diese sollte die Dienstleistungsvereinbarung zwischen den damaligen Generaldirektionen Außenbeziehungen (GD RELEX), Entwicklung und EuropeAid aus dem Jahr 2001 ersetzen und detaillierte Vorschriften für den Ablauf des Programmplanungs- und Verwaltungszyklus zwischen EAD und Kommission enthalten. Allerdings sollte es dann noch eineinhalb Jahre in Anspruch nehmen, bis die Modalitäten der Zusammenarbeit zwischen dem EAD und der neuen Generaldirektion ,EuropeAid Entwicklung und Zusammenarbeit‘ (GD DEVCO) schließlich am 13. Januar 2012 in einer dienstübergreifenden Vereinbarung fixiert werden konnten. ${ }^{27}$

Die Zeit, die diese Feinjustierung in Anspruch nahm, zeigte deutlich das Ausmaß des Klärungsbedarfs, auch wenn Ashton diesen in ihrem ersten EAD-Bericht im Dezember 2011 noch ausgeklammert und lediglich darauf hingewiesen hatte, dass EAD und Kommission bei den Vorbereitungen für den nächsten mehrjährigen Finanzrahmen eng zusammenarbeiten sollten. ${ }^{28}$ Dabei lagen die zentralen organisatorischen Streitpunkte gerade bei den drei wichtigsten Außenhilfeinstrumenten EEF, DCI und ENPI auf der Hand: Was würde es in der Praxis bedeuten, wenn, wie es in Art. 9 Abs. 4 EAD-Beschluss heißt, alle Vorschläge von den einschlägigen Dienststellen des EAD und der Kommission ,unter der Verantwortung“ des zuständigen Kommissionsmitglieds ,gemeinsam erstellt und gemeinsam mit dem Hohen Vertreter zur Annahme durch die Kommission vorgelegt“" würden? ${ }^{29}$ Welche Rollen sollten der EAD und die Kommissionsdienste bei der Ausarbeitung der Mittelzuweisungen, der Länder- und Regionalstrategiepapiere und der nationalen und regionalen Richtprogramme im Rahmen der wichtigsten Außenhilfeinstrumente übernehmen? Welcher Weisungsbefugnis würden die neuen EU-Delegationen unterliegen? Wie ließen sich die Verfahrensabläufe zwischen den neuen Diensteinheiten möglichst harmonisch und ohne Duplizierungen gestalten? Und wer sollte im Fall von Meinungsverschiedenheiten das letzte Wort haben?

26 Vgl. den gemeinsamen Brief von Catherine Ashton und Andris Piebalgs vom 27. Juli 2010 an Eva Joly, MdEP, auf ihre Anfrage den Entwurf des EAD-Gründungsbeschlusses betreffend, abrufbar unter: http://www.europar 1.europa.eu/meetdocs/2009_2014/documents/deve/dv/ashton-piebalgs_jo/ashton-piebalgs_joly.pdf (letzter Zugriff: 25.10.2012).

27 Am 13. Januar 2012 unterzeichneten der Generalsekretär der Kommission und der Chief Operating Officer des EAD eine dienstübergreifende Vereinbarung. Europäische Kommission: Working Arrangements between Commission Services and the European External Action Service (EEAS) in relation to external relations issues, SEC (2012) 48, unveröffentlichtes SEC-Dokument liegt der Autorin vor - im Folgenden dienstübergreifende Vereinbarung.

28 Sie kam damit der Forderung des Art. 13 Abs. 2 des EAD-Beschlusses nach, über die Erfahrungen mit den Kooperationsvereinbarungen zwischen dem EAD und den Kommissionsdienststellen hinsichtlich der Verwaltung der externen Finanzinstrumente, insbesondere der Programmierung der Hilfe, Bericht zu erstatten. Europäischer Auswärtiger Dienst: Report by the High Representative to the European Parliament, the Council and the Commission 22nd December 2011, hier Abschnitt 14.

29 Die Grundlagen der Außen- und Entwicklungshilfeinstrumente EEF und DCI (geografisch) und ENPI ähneln sich. Vgl. dazu Art. 9 Abs. 4 zur Entwicklungszusammenarbeit respektive Art. 9 Abs. 5 zur Nachbarschaftspolitik des EAD-Beschlusses. Hier wird nachfolgend vorrangig die Organisation für DCI und EEF dargestellt. 


\section{Entwicklung im EAD}

Als diplomatischer Dienst unterstützt der EAD als Gremium sui generis - bewusst nicht als EU-Institution, sondern als eine funktional unabhängige Einrichtung konzipiert - die Hohe Vertreterin..$^{30}$ Er ist unter anderem verantwortlich für den politischen Dialog und übernimmt die Verantwortung bei der Mittelvergabe und der Programmierung der Außen- und Entwicklungshilfe. Seine Aufgabe ist es, die Kohärenz der verschiedenen Dimensionen des Außenhandelns der Europäischen Union - etwa in den Bereichen Außen- und Sicherheitspolitik, Handel, Agrar- und Fischereipolitik, Umweltschutz, Entwicklungszusammenarbeit - sowie eine einheitliche Linie der Europäischen Union gegenüber Dritten sicherzustellen. Im Bereich der Außen- und Entwicklungshilfe soll der EAD sicherstellen, dass auch den Zielen der Entwicklungspolitik der Union nach Art. 208 AEUV Rechnung getragen wird. Neben der Armutsbekämpfung soll er die Verwirklichung der Ziele des Europäischen Konsenses über die Entwicklungspolitik und des Europäischen Konsenses über die humanitäre Hilfe fördern. ${ }^{31}$

Der seit Frühjahr 2012 am Rond-point Schuman residierende EAD setzt sich anteilig aus ehemaligem Personal der Europäischen Kommission, des Ratssekretariats sowie aus den EUMitgliedstaaten zusammen. In der Zentrale des EAD in Brüssel arbeiten rund 1.500 Beamte, hinzukommen die Mitarbeiter in den EU-Delegationen. Organigramme und Verfahrenscharts ersetzen nicht den Blick hinter die Kulissen, ablesen lassen sich aus ihnen jedoch die Umstrukturierungen rund um den EAD. ${ }^{32}$ So zeigt sich insbesondere der Transfer bestehender Arbeitseinheiten, der eine schnelle Arbeitsaufnahme des EAD erst ermöglicht hatte. Neben Einheiten aus dem Generalsekretariat des Rates (insbesondere der Policy Unit und der Generaldirektion E), ${ }^{33}$ wurden die gesamte Generaldirektion Auswärtige Beziehungen ${ }^{34}$ und beinahe einhundert, vor allem länder- und regionenbezogene Stellen aus der ehemaligen Generaldirektion Entwicklung in den EAD überführt. ${ }^{35}$

Gegliedert ist der EAD in fünf regionale Arbeitseinheiten (Asien und pazifischer Raum, Europa und Zentralasien, Naher Osten und die südliche Nachbarregion, Nord- und Südame-

30 Art. 27 EUV.

31 Dies wurde auf Druck des Europäischen Parlaments in Erwägungsgrund 4 EAD-Beschluss verankert. Dieser Einschub bezieht sich trotz des Verweises auf allgemeine Ziele letztlich jedoch ausdrücklich nur auf den Bereich der Entwicklungszusammenarbeit und damit nicht auf das gesamte Handeln des EAD.

32 Europäischer Auswärtiger Dienst: European External Action Service. Graphic representation, 1.5.2012, abrufbar unter: http://eeas.europa.eu/background/docs/organisation_en.pdf (letzter Zugriff: 1.5.2012).

$33 \mathrm{Zu}$ den in den EAD überführten Verwaltungseinheiten und Aufgabenbereichen siehe den Anhang des EADBeschlusses: Die Generaldirektion E wurde in ihrer Gesamtheit in den EAD überführt. Im Einzelnen waren dies die unmittelbar dem Generaldirektor unterstellten Referate, die Direktion ,Amerika und Vereinte Nationen', die Direktion ,Westlicher Balkan, Osteuropa und Zentralasien', die Direktion ,Nichtverbreitung von Massenvernichtungswaffen', die Direktion ,Parlamentarische Angelegenheiten im Bereich der GASP' und die Verbindungsbüros in New York und Genf.

$34 \mathrm{Zu}$ den in den EAD überführten Verwaltungseinheiten und Aufgabenbereichen siehe den Anhang des EADBeschlusses. Aus der ehemaligen Generaldirektion ,Auswärtige Beziehungen“ waren dies unter anderem alle Planstellen mit hierarchischer Funktion und das diesen unmittelbar beigeordnete Unterstützungspersonal, Direktion A ,Krisenplattform und Politikkoordinierung in der GASP', Direktion B ,Multilaterale Beziehungen und Menschenrechte', Direktion C ,Nordamerika, Ostasien, Australien, Neuseeland, EWR, EFTA, San Marino, Andorra und Monaco', Direktion D ,Koordinierung der Europäischen Nachbarschaftspolitik', Direktion E ,Osteuropa, Südlicher Kaukasus, Zentralasiatische Republiken', Direktion F ,Naher und Mittlerer Osten, Südlicher Mittelmeerraum', Direktion G ,Lateinamerika', Direktion H ,Asien, außer Japan und Korea'.

35 Anteilig wurden aus der GD RELEX 585 Stellen, aus der Generaldirektion Entwicklung 93 Stellen, aus den Delegationen der GD RELEX 436 Stellen und aus dem Rat 411 Stellen übertragen. Vgl. Europäische Kommission: Ein neuer Schritt beim Aufbau des EAD: Personalversetzung zum 1. Januar 2011, Rapid-Pressemitteilung, 21.12.2012, IP/10/1769. 


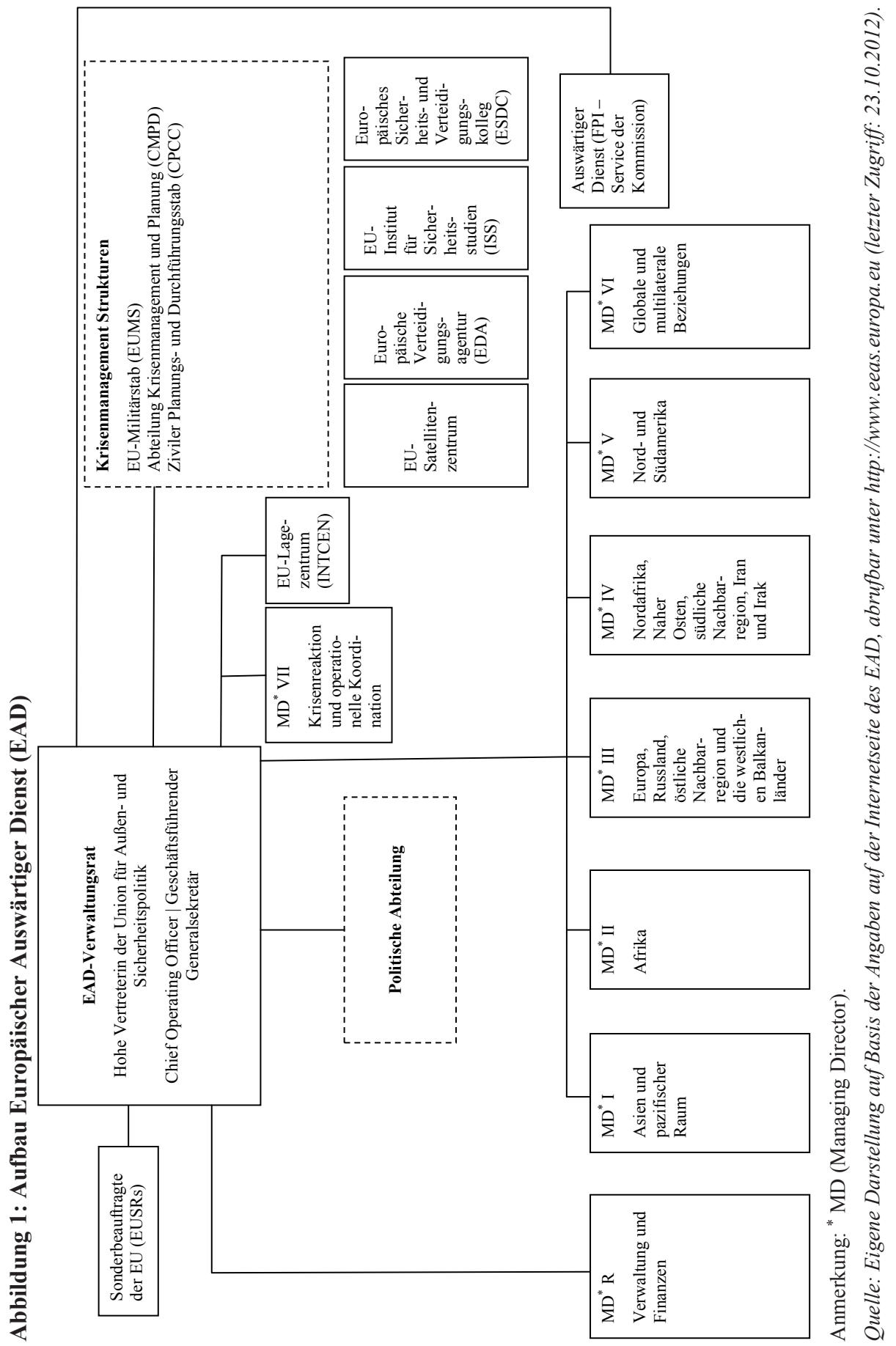


rika, Afrika), eine thematische Arbeitseinheit zu globalen und multilateralen Beziehungen sowie eine zu Personal- und Finanzfragen. Außerdem umfasst der EAD auch die Krisenmanagementstrukturen, die aus der Kommission und vor allem aus dem Ratssekretariat transferiert wurden. Die vormals bestehende Aufsplittung in die AKP-Staaten und die ,übrigen Staaten " wurde somit aufgelöst. Im EAD ist eine horizontale Einheit mit der Koordinierung der Entwicklungszusammenarbeit betraut, die innerhalb der Arbeitseinheit globale und multilaterale Beziehungen angesiedelt ist. Neben der Koordinierung der Entwicklungszusammenarbeit ist diese unter anderem auch zuständig für Menschenrechte und Demokratie, Wahlbeobachtung und Terrorismusbekämpfung. Die kleine Einheit dient als Anlaufstelle zu entwicklungspolitischen Themen. ${ }^{36} \mathrm{Zu}$ ihren Aufgaben gehört es, die Hohe Vertreterin sowohl bei der Vorbereitung von Entwicklungsthemen im Rat für Auswärtige Angelegenheiten zu unterstützen als auch in ihrer Funktion als Vizepräsidentin der Kommission, wenn entwicklungspolitische Themen im Kommissionskollegium auf der Agenda stehen. Hier sollen die Leitlinien für die Programmierung von EEF und DCI erarbeitet und für Kohärenz der Politik im Interesse der Entwicklungszusammenarbeit innerhalb des EAD gesorgt werden. $\mathrm{Zu}$ diesem Zweck arbeitet sie mit den entsprechenden thematischen und geografischen Direktionen zusammen und koordiniert die Eingaben des EAD für die Entwürfe der Kommissionsmitteilungen der GD DEVCO. Ihr obliegt es, die Taskforce ,Entwicklung' des EAD zu koordinieren, die sich aus Beamten der verschiedenen geografischen und thematischen Direktionen zusammensetzt und die einmal monatlich zusammentrifft. Angesichts dieses breiten Aufgabenspektrums wurde die knappe personelle Ausstattung von der Entwicklungszusammenarbeit nahestehenden Organisationen bereits mehrfach kritisiert und als Zeichen dafür gewertet, dass dem Thema Entwicklung im EAD nicht ausreichend Priorität beigemessen wird. ${ }^{37}$

\section{Die neue Generaldirektion EuropeAid - Entwicklung und Zusammenarbeit (GD DEVCO)}

Auch bei der GD DEVCO zeigen sich die Umstrukturierungen in der Post-Lissabon-Phase rund um die Errichtung des EAD deutlich. Begonnen hatte die vorgezogene Neuorganisation mit dem Amtsantritt der Barroso-II-Kommission, die im Februar 2010 ihre Arbeit aufnahm. Kommissionspräsident José Manuel Barroso hatte die Europäische Nachbarschaftspolitik (ENP), die bis dahin im Kompetenzbereich der für die Außenbeziehungen zuständigen Kommissarin Benita Ferrero-Waldner lag, dem neuen, für Erweiterung zuständigen Kommissar Štefan Füle zugewiesen. Diese Entscheidung wurde einerseits als nicht zwingend kritisiert, gleichzeitig jedoch als Bemühen um die Erhaltung maßgeblicher außenpolitischer Handlungsfelder für die ,Kernkommission“ gewertet. ${ }^{38}$ Teil dieser proaktiven Strategie der Statussicherung war auch der Neuzuschnitt einer Reihe von Kompetenzbereichen der Europäischen Kommissare, der mit einer inhaltlichen Überlappung der Portfolios verbunden war und der von Kritikern als , asset stripping ' gebrandmarkt wurde. ${ }^{39}$ Begründet dürfte dieser Schritt zudem in der Tatsache gewesen sein, dass Barroso im Kommissionskollegium als Streitschlichter das letzte Wort hat. ${ }^{40}$ Und dies unabhängig davon, dass Ashton mit ihrem ,Doppelhut‘ als Vizepräsidentin auch der Kommission angehört und die, selbst wenn formal

36 Vgl. hier und nachfolgend van Seters/Klavert: EU development cooperation after the Lisbon Treaty, 2011.

37 Exemplarisch ebenda.

38 Udo Diedrichs: Europäische Kommission, in: Werner Weidenfeld/Wolfgang Wessels (Hrsg.): Jahrbuch der Europäischen Integration 2010, S. 75-84, hier S. 82.

39 Euractiv.com: 'Difficult birth' awaits EU diplomatic service, 9.3.2010.

40 Art. 248 AEUV. 
keine Hierarchie unter den Kommissaren besteht, als Hohe Vertreterin die Aufgabe hat, die auswärtigen Politiken zu koordinieren. ${ }^{41}$ Barroso ist auch, sofern er selbst den Vorsitz übernimmt (was bisher stets der Fall war) und diesen nicht der Hohen Vertreterin überlassen sollte, derjenige, der die Tagesordnung der von ihm errichteten ,Gruppe der für Außenbeziehungen zuständigen Kommissare“ bestimmt. ${ }^{42}$ Um ein kohärenteres Erscheinungsbild in den Außenbeziehungen zu fördern, hatte Barroso außerdem ,seine` drei für Entwicklungszusammenarbeit, humanitäre Hilfe sowie Erweiterung und Nachbarschaft zuständigen Kommissare aufgefordert, eng mit dem EAD zusammenzuarbeiten. ${ }^{43}$

Wie dargestellt, wurden zusätzlich zum Transfer der GD RELEX in den EAD vor allem länder- und regionenbezogene Stellen aus der Generaldirektion Entwicklung in den EAD ausgegliedert. ${ }^{44}$ Die in der Europäischen Kommission verbliebenen Mitarbeiter der ehemaligen Generaldirektion Entwicklung bilden nun gemeinsam mit EuropeAid die neu gebildete GD DEVCO. ${ }^{45}$ Sie hat unter der Leitung von Andris Piebalgs zum Jahreswechsel 2011 ihre Arbeit aufgenommen. Die neue Organisationsstruktur besteht aus vier politischen und thematischen Direktionen (EU-Entwicklungszusammenarbeit; Qualität und Wirkung; Nachhaltiges Wachstum und Entwicklung; Menschliche und gesellschaftliche Entwicklung), vier geografischen Direktionen (Subsahara-Afrika und horizontale AKP-Angelegenheiten; Nachbarschaft; Lateinamerika und Karibik; Asien, Zentralasien, Mittlerer und Naher Osten/ Golfstaaten und Pazifik) und einer administrativen Direktion. Damit gehört auch in der Kommission die bisherige Aufsplittung entwicklungspolitischer Zuständigkeiten in die zwei Generaldirektionen, Entwicklung (die für die AKP-Staaten zuständig war) und Auswärtige Beziehungen (in deren Zuständigkeitsbereich alle anderen Staaten fielen), der Vergangenheit an. Innerhalb der GD DEVCO übernimmt die Direktion A ,EU-Entwicklungspolitik' eine führende Rolle. ${ }^{46}$ Sie formuliert den allgemeinen Orientierungsrahmen für die Ausrichtung der Politik der Generaldirektion, wobei der Aufgabenbereich angefangen beim Thema Budgethilfe über Politikkohärenz im Interesse von Entwicklung bis hin zur Wirksamkeit der Hilfen und Themen wie fragile Staaten und Krisenmanagement reicht. Hinzukommen die Vertretung in internationalen Gremien bei Entwicklungsfragen, die Koordinierung mit den

41 Art. 18 und 21 EUV.

42 Vgl. Europäische Kommission: Vademecum on working relations with the European External Action Service (EEAS), SEC (2011) 1636, Punkt 2.2.1.

43 Vgl. José Manuel Barroso: Mission letters from President Barroso to the Commissioners designate an Andris Piebalgs (27.11.2009), Štefan Füle (27.11.2009) und Kristalina Georgieva (27.1.2010), abrufbar unter: http://e c.europa.eu/commission_2010-2014/mission_letters/index_en.htm (letzter Zugriff: 25.10.2012).

$44 \mathrm{Zu}$ den in den EAD überführten Verwaltungseinheiten und Aufgabenbereichen siehe den Anhang des EADBeschlusses: Aus der Generaldirektion ,Entwicklung“ verbleiben in der Kommission lediglich Direktion D ,AKP II - West- und Zentralafrika, Karibik und ÜLG‘ mit Ausnahme der Taskforce überseeische Länder und Gebiete, Direktion E ,Horn von Afrika, Ostafrika und südliches Afrika, Indischer Ozean und Pazifik', das für die Programmierung zuständige Personal des Referats CI ,AKP I: Entwicklungshilfeprogrammierung und -verwaltung', das für panafrikanische Beziehungen zuständige Personal des Referats C2 ,Panafrikanische Fragen und Institutionen, Governance und Migration“ sowie alle Planstellen mit hierarchischer Funktion und das diesen unmittelbar beigeordnete Unterstützungspersonal.

45 Vgl. Europäische Kommission: Directorate General for Development and Cooperation - EuropeAid, Organigramm, 1.9.2012, abrufbar unter: http://ec.europa.eu/europeaid/who/documents/organigramme-devco_en.pdf (letzter Zugriff: 15.10.2012).

46 Zur Mission und den Aufgaben der einzelnen Referate siehe Europäische Kommission: Directorate General for Development and Cooperation - EuropeAid. Main missions of DEVCO Directorates \& Units, 3.8.2011, abrufbar unter: http://ec.europa.eu/europeaid/who/about/documents/dg-devlpmt-coop-europeaid-mission-statements. pdf (letzter Zugriff: 25.10.2012); Europäische Kommission: Directorate General for Development and Co-operation - EuropeAid. Management Plan 2012, abrufbar unter: http://ec.europa.eu/atwork/synthesis/amp/doc/de vco_mp.pdf (letzter Zugriff: 25.10.2012). 

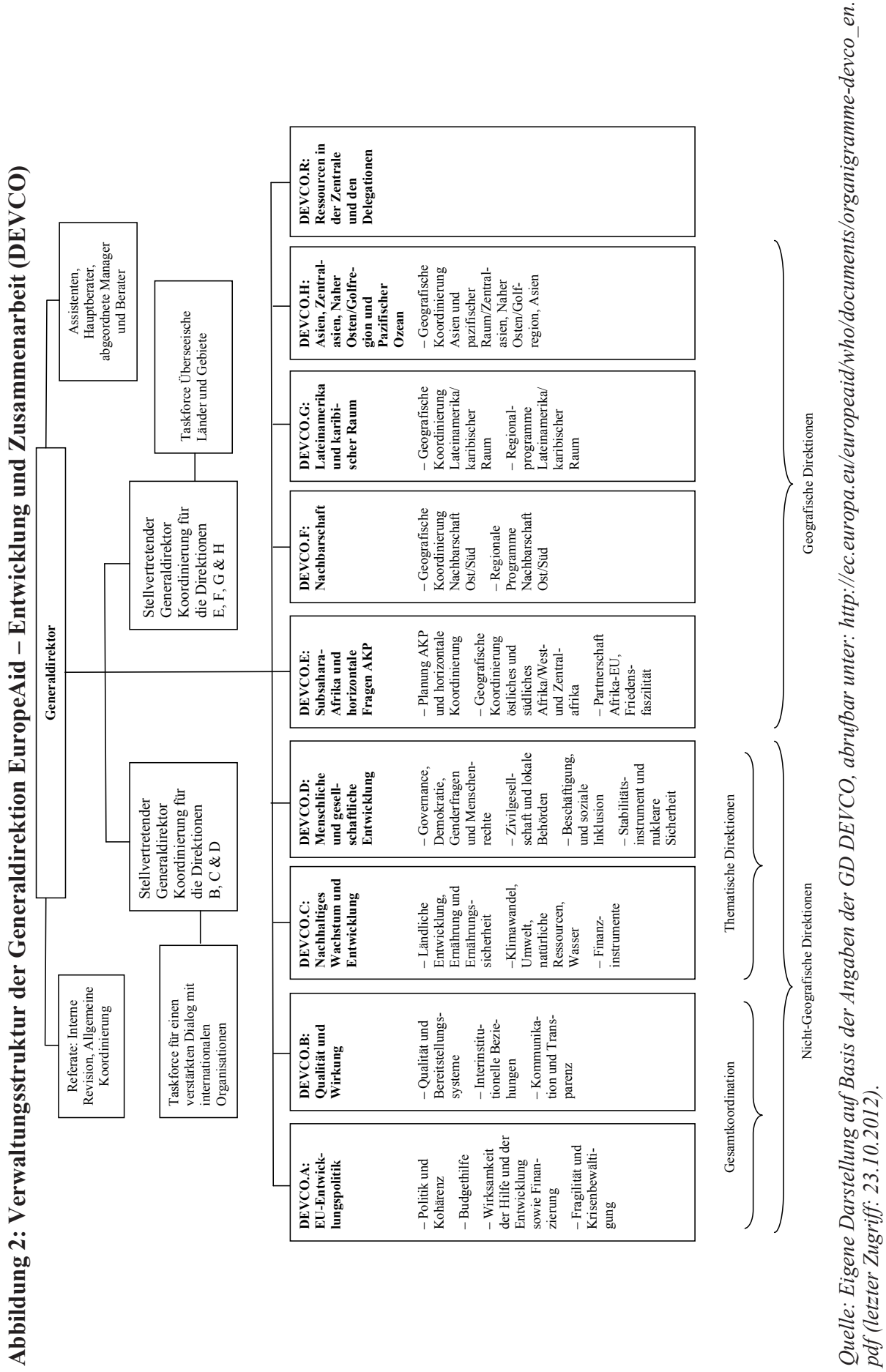
Mitgliedstaaten und Nicht-EU-Staaten sowie die Koordinierung an der Schnittstelle mit dem EAD im Bereich des Krisenmanagements.

In der Zentrale der GD DEVCO in Brüssel arbeiten zwischen 1.300 und 1.500 Mitarbeiter, hinzukommt das Personal in den EU-Delegationen. Zwar hat die GD DEVCO mit dem Transfer der länderbezogenen Beamten und Referaten selbst Personal an den EAD transferiert, gleichzeitig jedoch eine Reihe neuer Kompetenzen hinzugewonnen (etwa beim DCI, dessen geografische Programme bisher unter der Federführung der GD RELEX standen). Ursprünglich sollte die Überführung der gesamten GD RELEX in den EAD dazu dienen, eine Konkurrenz von EAD und der Hohen Vertreterin mit einem Europäischen Kommissar für Außenbeziehungen zu vermeiden ${ }^{47}$ Mit der aufgewerteten GD DEVCO ist nun dennoch ein gewichtiger Akteur entstanden und der für Entwicklung zuständige Kommissar Piebalgs vertritt zudem die Europäische Kommission im Rat Auswärtige Angelegenheiten und kann die Kommission auch in informellen Ratstreffen, wie den Gymnich-Treffen, repräsentieren. ${ }^{48}$ Hinzu kommt, dass die Kommission durch die in den EAD transferierten Kommissionsbeamten noch über einen verlängerten Arm in den EAD verfügt.

\section{Der Part der EU-Delegationen}

Mit dem Inkrafttreten des Vertrags von Lissabon am 1. Dezember 2009 wurden die 140 bestehenden Kommissionsdelegationen offiziell in EU-Delegationen umgewandelt. Die bisher eher technische Ausrichtung der Arbeit der Kommissionsdelegationen sollte mit den neuen EU-Delegationen politischer werden. Diese übernehmen nun auch auf die Gemeinsame Außen- und Sicherheitspolitik (GASP) bezogene Aufgaben der ehemals turnusmäßigen Präsidentschaft des Rates der Europäischen Union, wie beispielsweise den politischen Dia$\log .{ }^{49}$ Zusammengesetzt sind die Delegationen aus Mitarbeitern des EAD (die hauptsächlich mit politischen und repräsentativen Funktionen betraut sind) und der Kommission (die Durchführung und Evaluation derjenigen Maßnahmen übernehmen, die wie Entwicklungszusammenarbeit und Nachbarschaftspolitik in ihren Kompetenzbereich fallen) sowie beigeordnetem Personal aus den Mitgliedstaaten. Zwar unterstehen die Delegationen nach Art. 221 AEUV der Leitung der Hohen Vertreterin, allerdings können sowohl die Hohe Vertreterin, der EAD und - in Bereichen wie Entwicklungszusammenarbeit und Nachbarschaftspolitik ${ }^{50}$ - die Kommission direkte Anweisungen an die Delegation erteilen. Grundsätzlich sollen diese zwar durch den EAD ,gechannelt‘ werden, doch naturgemäß ergeben sich aus den mehrfachen Weisungsbefugnissen Schwierigkeiten, die durch den EAD und den Verbleib der Bereiche Nachbarschaftspolitik und Entwicklungszusammenarbeit in der GD DEVCO entstanden sind. Auf diese hat Ashton in ihrem ersten EAD-Bericht explizit hingewiesen und darin den Grundsatz bekräftigt, dass alle Delegationsmitarbeiter bei ihrer alläglichen Arbeit dem Delegationsleiter unterstehen, der notfalls bestimmte Angelegen-

47 Vgl. Sophie Vanhoonacker/Natasja Reslow: The European External Action Service: Living Forwards by Understanding Backwards, in: European Foreign Affairs Review 1/2010, S. 1-18, hier S. 9.

48 Vgl. Europäische Kommission: Vademecum on working relations, 2011, Punkt 3.3.

49 Vgl. zur Entstehungsgeschichte und den Rechtsgrundlagen der EU-Delegationen Edith Drieskens: What's in a Name? Challenges to the Creation of EU Delegations, in: The Hague Journal of Diplomacy 1/2012, S. 51-64; Steven Blockmans/Ramses A. Wessel (Hrsg.): Principles and practices of EU external representation: selected legal aspects, Centre for the Law of EU External Relations: CLEER Working Papers 2012/5.

50 Art. 5 Abs. 3 EAD-Beschluss. 
heiten auch an die Zentrale in Brüssel zurückverweisen kann (beispielsweise bei widersprüchlichen Weisungen des EAD und der Kommission). ${ }^{51}$

Der gemeinsame Beschluss von EAD und Kommission zu einem Kooperationsmechanismus soll das schwierige Verhältnis verbessern, indem mit diesem nicht nur die Position der Delegationsleiter bekräftigt, sondern auch ein gemeinsamer Lenkungsausschuss von EAD und GD DEVCO (EUDEL) eingerichtet wurde. ${ }^{52}$ Schließlich ist die koordinierende Rolle der EU-Delegationen gerade bei der Außen- und Entwicklungshilfe entscheidend. Sie übernehmen nicht nur eine wichtige Rolle in den verschiedenen Phasen der Strategie- und Programmplanung, sondern sind auch für die enge Zusammenarbeit mit den diplomatischen und konsularischen Vertretungen der Mitgliedstaaten - und damit das ,joint programming der Hilfen - zuständig. Gleichzeitig dienen sie als Anlaufstelle für die Partnerregierungen vor Ort und sind dort als „Augen und Ohren“53 für die Brüsseler Zentrale tätig.

\section{Die Programmplanung der Außen- und Entwicklungshilfe für den mehrjährigen Finanzrahmen 2014 bis 2020}

Mit dem EAD soll die Außen- und Entwicklungshilfe unter der Federführung des diplomatischen Dienstes außenpolitisch wirksamer und strategischer ausgerichtet werden. Vor der Vertragsreform von Lissabon war die Europäische Kommission für den gesamten fünfstufigen Programmierungszyklus zur Vergabe der Außen- und Entwicklungshilfe zuständig: Je nach geografischer Zuständigkeit erfolgten die ersten drei Programmierungsstufen entweder in der GD RELEX (DCI und ENPI) oder in der GD Entwicklung (EEF). Mit Ausnahme der thematischen DCI-Programme, ${ }^{54}$ die in den Verantwortungsbereich des Entwicklungskommissars fielen, trug der Kommissar für Außenbeziehungen dabei die politische Verantwortung. Die Durchführung wurde in den beiden anschließenden Programmierungsstufen von EuropeAid übernommen.

Mit dem EAD-Gründungsbeschluss werden diese Befugnisse im Programmierungszyklus neu verteilt, sodass nun die Entscheidungen der fünf Programmierungsphasen vom EAD (in der konzeptionellen, stärker politisch ausgerichteten Anfangsphase) und der Kommission (in der technischen Durchführungsphase) vorbereitet und implementiert werden sollen. Grundsätzlich soll demnach für die strategische Ausarbeitung der EU-Außenhilfeprogramme in erster Linie der EAD zuständig sein, während die Verwaltung der Programme für die Zusammenarbeit mit Drittstaaten in den Zuständigkeitsbereich der Kommission fällt. So trägt laut Ratsbeschluss der EAD zum Programmplanungs- und Verwaltungszyklus bei, indem er die Kommissionsbeschlüsse zu den strategischen, mehrjährigen Maßnahmen durch den Gesamtfinanzrahmen für die einzelnen Regionen, die Länder- und Regionalen Strategiepapiere sowie die Nationalen und Regionalen Richtprogramme vorbereitet. ${ }^{55}$

Ausgenommen davon ist allerdings - und das ist entscheidend - insbesondere die Planungshoheit für die strategisch wichtigen Bereiche der Nachbarschaftspolitik und der Entwicklungszusammenarbeit: Weiterhin „unter der Verantwortung“ - und nicht, wie es in

51 Vgl. Europäischer Auswärtiger Dienst: Report by the High Representative, 2011, Abschnitt 18.

52 Europäische Kommission/Hohe Vertreterin der Union für Außen- und Sicherheitspolitik: Joint Decision of the Commission and the High Representative of the Union for Foreign Affairs and Security Policy of 28.3.2012 on Cooperation Mechanisms concerning the Management of Delegations of the European Union, JOIN (2012) 8.

53 Furness: The Lisbon Treaty, 2012.

54 Die thematischen Programme machen rund 40 Prozent, die geografischen Programme rund 60 Prozent der im DCI bereitgestellten Mittel aus.

55 Art. 9 Abs. 3 EAD-Beschluss. 
Ashtons Entwurf noch hieß „under the guidance and supervision“ - der jeweils zuständigen Europäischen Kommissare verbleiben damit die drei wichtigsten Außen- und Entwicklungshilfeinstrumente DCI (die geografischen Programme), EEF und ENPI. Der Ratsbeschluss hält die Hohe Vertreterin und den EAD an, während des gesamten Zyklus der Programmplanung, bei Planung und Umsetzung mit den zuständigen Mitgliedern und Dienststellen der Kommission zusammenzuarbeiten. Alle Vorschläge für Beschlüsse sollen nach den Arbeitsverfahren der Kommission ausgearbeitet und der Kommission zur Annahme vorgelegt werden. ${ }^{56}$

Von den thematischen Programmen fallen alle - außer den Instrumenten für Demokratie und Menschenrechte, Nuklearsicherheit und, mit Einschränkungen, Stabilität - ausschließlich in die Zuständigkeit der neuen Generaldirektion. Dort sollen sie von den geeigneten Kommissionsdienststellen unter der Leitung des für Entwicklungspolitik zuständigen Kommissionsmitglieds vorbereitet und dem Kommissionskollegium in Absprache mit dem Hohen Vertreter und den anderen relevanten Kommissionsmitgliedern vorgelegt werden. ${ }^{57}$

Soweit sind die Vorgaben des EAD-Gründungsbeschlusses wenig konkret. Was bedeutet es aber nun in der Praxis, wenn alle Vorschläge von EAD und Kommission „,unter der Verantwortung“ des Entwicklungskommissars „gemeinsam erstellt“ und dem Kommissionskollegium gemeinsam mit dem Hohen Vertreter vorgelegt werden? Diese Frage sollte, wie von Ashton und Piebalgs angekündigt, in der Intervallphase zwischen dem Gründungsbeschluss und der dienstübergreifenden Vereinbarung beantwortet werden. Angesichts der Budgetverhandlungen der Außen- und Entwicklungshilfe für den mehrjährigen Finanzrahmen 2014 bis 2020 geriet diese Feinjustierung unter erheblichen Zeitdruck. Bereits Anfang 2012 sollte schließlich damit begonnen werden, die Höhe der länderbezogenen Mittelzuweisungen des EEF und DCI zur Festlegung des Gesamtfinanzrahmens zu bestimmen, sodass 2013 dann die entsprechenden Projekte und Programme entwickelt werden können und die Implementierung ab 2014 erfolgen kann.

56 Art. 9 Abs. 4 EAD-Beschluss.

57 Ebenda. 


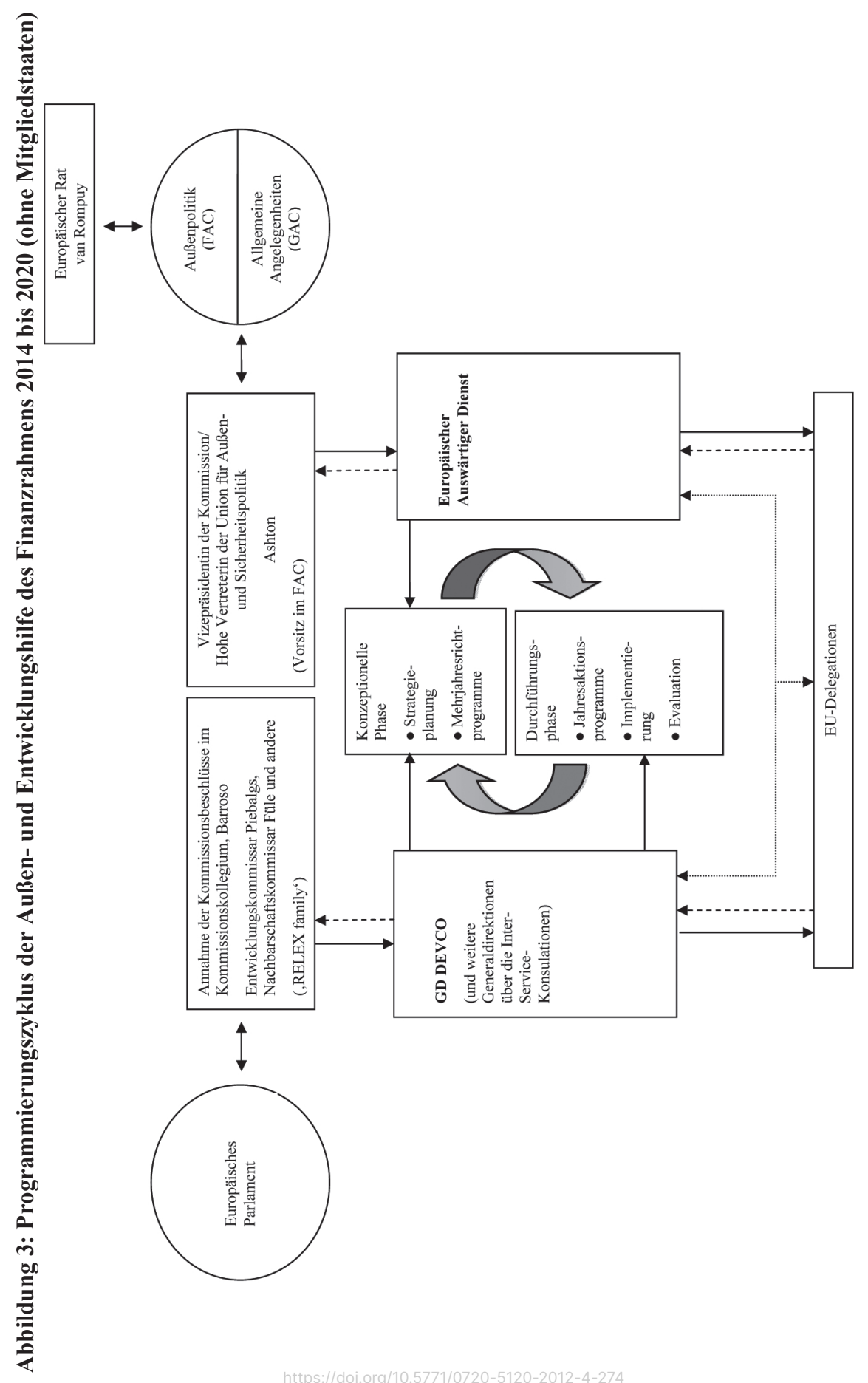

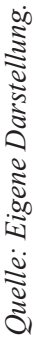


Tabelle 1: Die avisierten Mittel der geografischen und thematischen Außenhilfeinstrumente für den mehrjährigen Finanzrahmen 2014 bis 2020

\begin{tabular}{|c|c|c|c|}
\hline $\begin{array}{l}\text { Zuständigkeit für } \\
\text { die strategische } \\
\text { Ausrichtung }\end{array}$ & Instrument & $\begin{array}{c}\text { Mittel } \\
\text { (jeweilige Preise) }\end{array}$ & Ausrichtung \\
\hline \multirow{3}{*}{ EAD } & $\begin{array}{l}\text { Europäisches Instrument für Demokra- } \\
\text { tie und Menschenrechte (EIDHR) }\end{array}$ & 1.578 Mio. Euro & Thematisch \\
\hline & Stabilitätsinstrument (IfS) & 2.829 Mio. Euro & Thematisch \\
\hline & $\begin{array}{l}\text { Instrument für Zusammenarbeit im Be- } \\
\text { reich der nuklearen Sicherheit (INSC) }\end{array}$ & 631 Mio. Euro & Thematisch \\
\hline \multirow{4}{*}{$\begin{array}{l}\text { Gemeinsame Ver- } \\
\text { antwortung von EAD } \\
\text { und Kommission }\end{array}$} & Partnerschaftsinstrument (PI) & 1.131 Mio. Euro & Geografisch \\
\hline & $\begin{array}{l}\text { Europäisches Nachbarschaftsinstru- } \\
\text { ment (ENI) } \\
\text { - Nachbarschaftsstaaten der Östlichen } \\
\text { Partnerschaft und im Mittelmeer- } \\
\text { raum }\end{array}$ & 18.182 Mio. Euro & Geografisch \\
\hline & $\begin{array}{l}\text { Europäischer Entwicklungsfonds } \\
\text { (EEF), außerhalb des Haushaltsplans } \\
\text { - AKP-Staaten }\end{array}$ & 34.276 Mio. Euro & Geografisch \\
\hline & $\begin{array}{l}\text { Finanzierungsinstrument für die Ent- } \\
\text { wicklungszusammenarbeit (DCI) } \\
\text { - Länder Asiens, Lateinamerikas, Na- } \\
\text { her Osten, Zentralasien }\end{array}$ & $\begin{array}{l}\text { insgesamt } \\
\text { 23.295 Mio. Euro, } \\
\text { davon: } \\
\text { 13.991,5 Mio. Euro }\end{array}$ & Geografisch \\
\hline \multirow{3}{*}{ Kommission } & $\begin{array}{l}\text { Thematische Programme (Instru- } \\
\text { ment für globale öffentliche Güter } \\
\text { und Herausforderungen; Instrument } \\
\text { für nicht-staatliche Akteure und lo- } \\
\text { kale Behörden; Pan-Afrikanisches } \\
\text { Programm) }\end{array}$ & 9.303,2 Mio. Euro & Thematisch \\
\hline & $\begin{array}{l}\text { Instrument für Heranführungshilfe } \\
\text { (IPA) } \\
\text { - Beitrittskandidaten, Island, Türkei }\end{array}$ & 14.110 Mio. Euro & Geografisch \\
\hline & $\begin{array}{l}\text { Instrument für Grönland, fällt nicht un- } \\
\text { ter die allgemeine Durchführungsver- } \\
\text { ordnung }\end{array}$ & 219 Mio. Euro & Geografisch \\
\hline
\end{tabular}

Quelle: Eigene Zusammenstellung auf Basis der Höhe der bereitzustellenden Mittel entsprechend der Kommissionsvorschläge für den mehrjährigen Finanzrahmen. Vgl. Europäische Kommission: Multiannual Financial Framework 2014-2020. Strengthening Europe's place in the world, 17.2.2012, abrufbar unter: http://ec.europa.eu/europeaid/how/finance/mff/financial_framework_news_en.htm (letzter Zugriff: 24.10.2012). 
Mit den Post-Lissabon-Neuerungen wird, wie schließlich in der dienstübergreifenden Vereinbarung vom Januar 2012 fixiert werden konnte, dem ohnehin bereits kleinteiligen Prozess noch eine weitere Schicht an Komplexität hinzugefügt: ${ }^{58} \mathrm{Zu}$ den Aufgaben, die sich EAD und Kommission bei den Beschlüssen zu EEF und den geografischen Programmen des DCI (die thematischen Programme werden unter Verantwortung der Kommission verabschiedet) teilen, zählen insbesondere die Vorbereitung der Mittelzuweisungen sowie die Ausarbeitung der Strategiepapiere sowie der Richtprogramme. Wie auch bei der Strategieund Programmplanung vor dem Inkrafttreten des Vertrags von Lissabon, als diese noch arbeitsteilig zwischen GD RELEX, GD Entwicklung und den Kommissionsdelegationen erfolgte, übernehmen die EU-Delegationen dabei einen wichtigen Part.

So ist in der dienstübergreifenden Vereinbarung vorgesehen, dass der EAD, im Einvernehmen mit der GD DEVCO, die Vorgaben des Entwicklungskommissars für den Programmierungsprozess an die EU-Delegationen vorbereitet. Auf deren Grundlage beginnen diese den Konsultationsprozess mit den Partnerstaaten und senden im Anschluss ihre Beurteilungen mit den Entwürfen der Strategiepapiere und Richtprogramme an den EAD und die GD DEVCO. Der EAD reicht diese an die relevanten Kommissionsdienststellen und die entsprechenden Dienststellen, die in die Country Team Meetings (CTM) einbezogen sind, weiter und organisiert, gemeinsam mit der GD DEVCO, die CTM, zu denen auch andere relevante Kommissionsdienststellen hinzugezogen werden können. Die CTM sind für die Kohärenz der Programmierungsvorgaben zuständig und prüfen die Entwürfe im weiteren Austausch mit den EU-Delegationen. Auch in die nachfolgenden Schritte, wie den InterserviceKonsultationen mit allen Kommissionsdienststellen (die wiederum vom EAD initiiert werden) und dem Komitologieverfahren, sind der EAD und im Fall des ,joint programming ‘ auch die Mitgliedstaaten (unter dem Vorsitz der GD DEVCO) eingebunden. Die Entwürfe werden vom EAD im Einvernehmen mit der GD DEVCO ausgearbeitet und der EAD ist eingeladen, die Entwürfe der Kommissionsbeschlüsse den Komitologieausschüssen vorzustellen. Gegenüber dem Europäischen Parlament sind schließlich sowohl die GD DEVCO als auch der EAD zu den Entwürfen der Kommissionsbeschlüsse Rede und Antwort schuldig (dies ist nicht der Fall für den EEF, da dieser außerhalb des EU-Haushalts steht). Die Annahme der Kommissionsbeschlüsse im Kommissionskollegium erfolgt nach Initiierung durch den EAD gemäß den regulären Kommissionsverfahren. Ist diese erfolgt, beginnt die GD DEVCO mit der Ausarbeitung der jährlichen Aktionsprogramme für die Implementierung der Kommissionsbeschlüsse.

Bereits diese vereinfachte Darstellung veranschaulicht, dass mit der Ablösung der GD RELEX durch den EAD die Verfahren keineswegs wie intendiert entschlackt, sondern stattdessen schwerfälliger konstruiert wurden. Für deren Funktionieren ist nun ein Arbeitsfluss notwendig, bei dem die beiden Dienste in enger Zusammenarbeit mit den EU-Delegationen als quasi-organische Einheit zusammenarbeiten müssen. Angesichts der institutionellen

58 Vgl. die dienstübergreifende Vereinbarung zu den Programmierungsarrangements zu ENPI, DCI und EDF Abschnitt 3, zu den Länder- und Regionalen Strategiepapieren und zu den mehrjährigen Richtprogrammen insbesondere Punkt 3.4.2 sowie zu den thematischen DCI-Programmen Punkt 3.4.3. Für Letzteren ist das Verfahren weitgehend spiegelverkehrt geregelt: Vgl. dazu insbesondere auch Simone Görtz/Niels Keijzer: Reprogramming EU development cooperation for 2014-2020. Key moments for partner countries, EU Delegations, member states and headquarters in 2012, European Centre for Development Policy Management: ECDPM Discussion Paper 129, April 2012. 
Vorgeschichte ist dies, wie sich bereits zeigte, zunächst einmal zumindest fragwürdig. ${ }^{59}$ Daran ändert auch die Tatsache wenig, dass die Europäische Union neben innovativeren Finanzinstrumenten mit den differenzierten Entwicklungspartnerschaften eine Reihe von Neuerungen bei der Mittelvergabe für den nächsten mehrjährigen Finanzrahmen 2014 bis 2020 einführt. Zu diesen zählen beispielsweise eine bessere Evaluierung des Bedarfs des Empfängerstaats und dessen Absorptionsfähigkeit, die Einführung einer gegenseitigen Rechenschaftspflicht sowie die Intention, EU-Maßnahmen in jedem Staat künftig auf höchstens drei Bereiche zu konzentrieren. ${ }^{60}$

\section{Folgen für die künftige Ausrichtung des Außen- und Entwicklungshilfe- instrumentariums}

Ashton hatte in ihrer Rede vor dem Europäischen Parlament eine gemeinsame, einheitliche Strategie als raison d'être des EAD entworfen. Ursprünglich sollte mit dem EAD alles anders - und damit besser - werden. Betrachtet man nach zwei Jahren der Umorganisation europäischer Verfahrensabläufe und der Neuordnung von Befugnissen die Strategie- und Programmplanung der Außen- und Entwicklungshilfe im Vergleich zur Situation vor dem Inkrafttreten des Vertrags von Lissabon, so muss man zu einem wenig zufriedenstellenden Urteil kommen. Dabei hatte gerade die Programmplanung mit den Reformen von Lissabon unter einer gemeinsamen Strategie gebündelter, einheitlicher und zielgerichteter erfolgen sollen. Anstelle eines kohärenten Vorgehens stellt die heutige Organisation der strategischen Programmierung der Außen- und Entwicklungshilfe zwischen EAD und Kommission bisher jedenfalls einen verschärften Mikrokosmos der Gesamtsituation vor dem Inkrafttreten des Vertrags von Lissabon dar. Und es stellt sich die Frage, ob mit der Errichtung des EAD nicht sogar das Gegenteil dessen bewirkt wurde, was ursprünglich beabsichtigt war und - selbst wenn man die Anfangsschwierigkeiten substrahiert - die bekannten Schwierigkeiten nicht in den neuen Arrangements sogar noch festgezurrt wurden.

In der Tat lässt sich der EAD nur in einem begrenzten Maße als neuartiger Akteur bezeichnen. Viele Unzulänglichkeiten erklären sich sowohl aus seiner 40-jährigen Vorgeschichte als auch aus den grundlegenden Defiziten der außenpolitischen Gestaltungsmacht Europas. ${ }^{61}$ Doch auch eine inkrementale Fortschreibung im Rahmen des politisch Gangbaren, so wie es bei der Schaffung des EAD beobachtet werden konnte, beeinflusst den künftigen Kurs der EU-Außenbeziehungen. So spiegeln sich die kleinteiligen Arrangements vor der Reform durch den Vertrag von Lissabon bereits in dem Kompromiss des Quadrilogs zum EAD und ebenso in der nachträglichen Feinjustierung des EAD-Gründungsbeschlusses wider. Dies ist insbesondere der Fall durch den Transfer bestehender Arbeitseinheiten en bloc

59 Vereinfacht werden soll dieses Verfahren, indem die zunehmend als rigide wahrgenommenen siebenjährigen Länder- und Regionalen Strategiepapiere soweit möglich durch die neuen, sogenannten Joint Framework Documents (JFD), nationale und regionale Entwicklungspläne der Partnerstaaten oder durch gemeinsam mit den EU-Mitgliedstaaten erstellte Programmierungsdokumente ersetzt werden und entsprechend auch diese neuen Strategieplanungsdokumente als Grundlage für die Ausarbeitung der mehrjährigen Richtprogramme sowie Jahresaktionsprogramme dienen können.Vgl. Europäische Kommission/Hohe Vertreterin der Union für Außenund Sicherheitspolitik: Gemeinsame Mitteilung an das Europäische Parlament und den Rat. Globales Europa: Ein neues Konzept für die Finanzierung des auswärtigen Handelns der EU, KOM (2011) 865, S. 7.

60 Vgl. ebenda sowie Rat der Europäischen Union: Council conclusions 'Increasing the Impact of EU Development Policy: an Agenda for Change', $3166^{\text {th }}$ Foreign Affairs Council meeting, 14.5.2012.

61 Vgl. beispielsweise Vanhoonacker/Reslow: The European External Action Service, 2010; Ulrike Guérot: Der Wert Europas und seine Grenzen: die EU als außenpolitische Gestaltungsmacht mit Defiziten, in: integration 2/2012, S. 116-122. 
in den EAD und durch die Adaption der komplexen Arbeitsabläufe der Programmplanung, die die bisherige Zuständigkeitsverteilung von GD RELEX, GD Entwicklung und EuropeAid duplizieren. Selbst wenn die zentralen Bereiche Entwicklung und Nachbarschaft in der Verantwortung der Kommission verbleiben - und damit zunächst einmal dem unmittelbaren politischen Einfluss des EAD und eines wie auch immer gearteten ganzheitlichen Ansatzes entzogen sind - lässt sich feststellen, dass zwei bereits vor dem Inkrafttreten des Vertrags von Lissabon vorhandene Konfliktlinien nun deutlicher hervortreten:

Erstens wird die Armutsbekämpfung künftig deutlicher propagiert werden. Bereits in den letzten Jahren wurde wiederholt kritisiert, dass ein hoher Anteil an ODA-Mitteln an Staaten mit mittlerem Einkommen vergeben wird, statt diese den ärmsten Staaten zukommen zu lassen, was, so wurde argumentiert, der Armutsbekämpfung im Wege steht. Diese Argumentationslinie verlief bisher auch innerhalb der Kommission zwischen der GD RELEX und der GD Entwicklung und ist durch die Ausgliederung der GD RELEX in den EAD nun sichtbarer geworden. In der institutionellen Selbstbehauptung gegenüber dem EAD nutzt die GD DEVCO als achtundzwanzigster Akteur auf der globalen Entwicklungsagenda vermehrt die Armutsbekämpfung zur Profilschärfung und legitimiert so gleichzeitig ihren politischen Auftrag. In der Agenda für Wandel und bei der Neuausrichtung der Nachbarschaftspolitik $^{62}$ ist dies vorerst programmatisch sichtbar geworden und fließt in die gegenwärtige Mittelallokation für den Finanzrahmen 2014 bis 2020 ein. ${ }^{63}$ So soll beispielsweise im Falle des DCI bei der Vergabe der Mittel prinzipiell der ,Grundsatz der Differenzierung' greifen, um künftig sicherzustellen, dass die Hilfen auch bei den ärmsten Staaten beziehungsweise in Staaten mit mittleren Einkommensstrukturen bei den bedürftigsten Bevölkerungsschichten ankommen. Für Staaten mit mittleren Einkommensstrukturen (wie etwa den BRICS-Staaten) werden mit dem neuen Partnerschaftsinstrument andere Formen der Zusammenarbeit geschaffen.

Zweitens ist der Nexus von Sicherheit und Entwicklung ebenfalls sichtbarer geworden. In den letzten Jahren wurde dieser mit der Bereitstellung auch zivil-militärischer Krisenmanagementkapazitäten und dem Aufkommen des ganzheitlichen Ansatzes in den EU-Außenbeziehungen von einer vielfach befürchteten ,Versicherheitlichung' begleitet. Befürchtet wurde, dass ein solcher Ansatz auf Kosten der Armutsbekämpfung eine Unterordnung genuin entwicklungspolitischer Ziele unter ein sicherheitspolitisch dominiertes Krisenmanagement mit sich bringen wird. Und Ashton hatte tatsächlich frühzeitig deutlich gemacht, dass sie ihren Auftrag ernst nehmen und auch die Einbindung der Entwicklungspolitik in die außenpolitische Zielsetzung erleichtern würde. In der Entwicklungsgemeinschaft hat sich inzwischen die Logik durchsetzen können, dass die Gewährleistung von Sicherheit in den Drittstaaten eine der Voraussetzungen für den Erfolg entwicklungspolitischer Maßnahmen ist,

62 Europäische Kommission/Hohe Vertreterin der Union für Außen- und Sicherheitspolitik: Gemeinsame Mitteilung an das Europäische Parlament, den Rat, den Europäischen Wirtschafts- und Sozialausschuss und den Ausschuss der Regionen. Eine neue Antwort auf eine Nachbarschaft im Wandel, KOM (2011) 303; Barbara Lippert weist darauf hin, dass sich in der Gemeinsamen Mitteilung von Catherine Ashton und Stefan Füle zur revidierten ENP nur wenig Anzeichen für ein stärkeres außenpolitisches Engagement finden. Vgl. Barbara Lippert: Grenzwertig: die Fortsetzung der EU-Erweiterung als Stabilitätsexport, in: integration 2/2012, S. $123-128$.

63 Signifikante Neuerungen sind in dem Legislativpaket nicht enthalten, wobei eine Reihe von Änderungen diskutiert wird. Im Gegensatz zu den umfangreichen Reformen der Außenhilfeinstrumente von 2006, für die zwei Jahre intensiver interinstitutioneller Verhandlungen nötig waren, gestalten sich die Budgetverhandlungen für den mehrjährigen Finanzrahmen 2014 bis 2020 bisher weit weniger kontrovers. Vgl. Sandra Bartelt: The Institutional Interplay Regarding the New Architecture for the EC's External Assistance, in: European Law Journal 5/2008, S. 655-679. 
sodass sich in den letzten Jahren eine Reihe von Schnittflächen ergeben hat. Prominente Beispiele aus der Arbeit des EAD zeigen aber auch, dass die Entwicklungszusammenarbeit zudem zunehmend als ein für die Sicherheit Europas relevantes Thema wahrgenommen wird. Dies illustrieren etwa die Strategie für das Horn von Afrika ${ }^{64}$ und insbesondere die Strategie für Entwicklung und Sicherheit in der Sahel-Region 2011. ${ }^{65}$ Die europäische Entwicklungszusammenarbeit wird zunehmend politischer diskutiert und mit Themen wie Piraterie, Unruhen in den EU-Grenzregionen und Migrationsthemen offensiver auch mit der eigenen Sicherheit verknüpft.

Es kann also davon ausgegangen werden, dass künftig sowohl die Debatten um den Nexus von Sicherheit und Entwicklung intensiver geführt werden und die Armutsbekämpfung auch bei der Evaluierung der Wirksamkeit des Mitteleinsatzes stärker in den Vordergrund rücken wird. Mit tektonischen Verschiebungen bei der Ausrichtung der EU-Außenbeziehungen ist jedoch durch die Reorganisation der Verfahrensabläufe und der damit einhergehenden institutionellen Distanz zwischen den ehemaligen Arbeitsbereichen der GD RELEX und der GD Entwicklung nicht zu rechnen.

Selbst wenn sich frühzeitig abzuzeichnen schien, dass durch die Komplexität des gemeinsamen Programmierungszyklus insbesondere der drei finanziell am besten ausgestatteten Instrumente EEF, DCI und ENPI Streitigkeiten vorprogrammiert sein würden, hat die dienstübergreifende Zusammenarbeit auf der Arbeitsebene bisher - im positiven wie im negativen Sinn - keine größeren Überraschungen bereit gehalten. Durch den Transfer bestehender Arbeitseinheiten kennen sich die Beamten untereinander beziehungsweise sind in der Mehrzahl mit den bisherigen Kommissionsverfahren vertraut. Entsprechend ist durchaus eine gewisse dienstübergreifende Fraternisierung auf den Arbeitsebenen sichtbar, während vor allem auf politischer Ebene die alten Dispute ausgetragen werden. Weit problematischer zeigt sich die Situation hingegen in den EU-Delegationen, wo die Duplizierungen direkter und im Alltagsgeschäft spürbarer sind. Dies ist etwa der Fall, wenn es zu Loyalitätskonflikten bei Weisungen und Berichtspflichten gegenüber dem EAD beziehungsweise der Kommission kommt. Schon heute ist absehbar, dass dies nicht nur die ersten Phasen der Strategieund Programmplanung in den Jahren 2012 bis 2013 betrifft. Soll die angekündigte Flexibilisierung und Differenzierung des Entwicklungs- und Außenhilfeinstrumentariums auch umgesetzt und die Implementierung in den Jahren 2014 bis 2020 tatsächlich auch außenpolitisch wirksamer erfolgen, sind ein intensiver Austausch und eine enge Zusammenarbeit auf allen Arbeitsebenen weiterhin unabdingbar. Ohne eine Lösung der systembedingten Hürden bei der Organisation der Außen- und Entwicklungshilfe wird dies von den unteren Arbeitsebenen vor Ort kaum allein zu bewältigen sein.

\section{Fazit}

Zusammenfassend lässt sich feststellen, dass gerade für den viel beschworenen ganzheitlichen Ansatz in den EU-Außenbeziehungen die skizzierte Kleinteiligkeit und Schwerfälligkeit der neuen Verfahren im Bereich der Außen- und Entwicklungshilfe keinen erkennbaren Mehrwert bringt. Daran wird auch die Tatsache wenig ändern, dass dem gesamten Bereich Entwicklung und den damit verbundenen Themen wie Menschenrechte, Demokratieförderung und Rechtsstaatlichkeit in den EU-Außenbeziehungen sowie ihrer Perzeption

64 Rat der Europäischen Union: Horn von Afrika - Schlussfolgerungen des Rates, Dok. 16858/11.

65 Europäischer Auswärtiger Dienst: Strategy for Security and Development in the Sahel, abrufbar unter: http://eeas.europa.eu/africa/docs/sahel_strategy_en.pdf (letzter Zugriff: 25.10.2012). 
in der sie begleitenden Forschung künftig wieder eine größere Bedeutung beigemessen werden wird. Maßgeblich zu der Aufwertung dieser, weichen' Themen beigetragen haben die unrühmliche Rolle der Europäischen Union und ihrer Mitgliedstaaten bei den Umbrüchen im Nahen und Mittleren Osten, der relative Bedeutungsverlust von GASP und GSVP angesichts potenzieller Trends einer (Re-)Nationalisierung in der Außen- und Sicherheitspolitik sowie der Omnipräsenz des währungspolitischen Krisenmanagements auf der europäischen Bühne. Unter diesen Vorzeichen ist es der Europäischen Kommission in ihrer Selbstbehauptung gegenüber dem EAD gelungen, als Konstante auf der europäischen Bühne zu agieren. Mit ihrer Agenda für Wandel und den Impulsen für eine Anpassung der Nachbarschaftspolitik hat sie das politische Fahrtenbuch für das europäische Außen- und Entwicklungshilfeinstrumentarium vorgegeben. Auf ihre Initiative hin ist die Europäische Union bei der Neuaufstellung ihres Außen- und Entwicklungshilfeinstrumentariums für die Jahre nach 2013 im Begriff, zumindest auf deklaratorischer Ebene ein neues Kapitel aufzuschlagen. Der EAD wird seinerseits darum bemüht sein, über seine Mitwirkung in den gemeinsamen Arrangements hinaus eigene außenwirksame Akzente zu setzen und insbesondere auch seinen ureigenen Part im Bereich Sicherheit offensiver auszuspielen.

Noch ist das letzte Wort bei der institutionellen Ausbalancierung der Zuständigkeiten für die Außen- und Entwicklungshilfe nicht gesprochen. Dies zeigen auch die Vorschläge der Gruppe zur Zukunft Europas um den deutschen Außenminister Guido Westerwelle. So fordern die elf Außenminister dieser sogenannten Zukunftsgruppe, die Nachbarschaftspolitik in den Zuständigkeitsbereich der Hohen Vertreterin zu verlagern und auch ihre Kompetenzen in der Entwicklungszusammenarbeit zu stärken. ${ }^{66}$ Es ist entsprechend davon auszugehen, dass bei der 2013 anstehenden Überprüfung des sich weiterhin im Aufbau befindlichen EAD die Organisation der Außen- und Entwicklungshilfe ein zentrales Anliegen bilden wird. Die sich daraus ergebenden Grundsatzfragen werden das Zusammenspiel von EAD und GD DEVCO sowie der EU-Delegationen als quasi-organische Einheit weiterhin erschweren.

$\mathrm{Ob}$ es nach beinahe zwei Jahren der organisatorischen Nabelschau gelingen wird, die vollmundigen Ankündigungen für den künftigen mehrjährigen Finanzrahmen auch tatsächlich umzusetzen, wird indes nicht allein von der dienstübergreifenden Harmonie zwischen EAD und Kommission abhängen. Damit gerade die Armutsbekämpfung kein Partikularinteresse im institutionellen Gefüge der Europäischen Union bleibt, ist weiterhin der Stellenwert entscheidend, der der Entwicklung im gesamten Kanon des auswärtigen Handelns eingeräumt wird. Die im Sinne der Politikkohärenz für Entwicklung maßgeblichen Bereiche wie insbesondere die Außenwirtschaftsbeziehungen, die Agrar- und Fischereisubventionen oder die Energie- und Klimapolitik - liegen in erster Linie außerhalb der Reichweite des EAD und stattdessen in den Händen der Europäischen Kommission. Aus dieser Perspektive betrachtet, schrumpfen die mit einer organisatorischen Harmonie von EAD und Kommission verknüpften Hoffnungen schnell auf ein reales Maß.

66 Abschlussbericht der Gruppe zur Zukunft Europas der Außenminister Belgiens, Dänemarks, Deutschlands, Frankreichs, Italiens, Luxemburgs, der Niederlande, Österreichs, Polens, Portugals und Spaniens, Warschau, 17.9.2012, S. 8-9, abrufbar unter: http://www.auswaertiges-amt.de/DE/Europa/Aktuell/120918_Zukunftsgrup pe_Warschau.html (letzter Zugriff: 25.10.2012). 